\title{
Diversidad genética en humanos mediante polimorfismos de inserción de Alu en la población de San Pelayo, Córdoba (Colombia)
}

\author{
Genetic diversity in humans through Alu insertion polymorphisms in \\ the population of San Pelayo, Córdoba- Colombia
Diversidade genética em humanos através de polimorfismos de inserção de Alu na população de San Pelayo, Córdoba- Colombia

\author{
Enrique Pardo Pérez ${ }^{\mathrm{a},}{ }^{*}$ Teodora Inés Cavadía Martínez ${ }^{\mathrm{b}}$ |Andrea Estefanía Cruz Cantero ${ }^{\mathrm{c}}$ \\ ${ }^{a}$ http://orcid.org/0000-0002-6467-5790

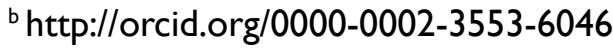 \\ c http://orcid.org/0000-000 I-57/7-47/2 \\ Universidad de Córdoba, Montería, Colombia
}

- Fecha de recepción: 2019-03-10

- Fecha concepto de evaluación: 2019-10-21

- Fecha de aprobación: 2019-10-28 http://dx.doi.org/ | 0.22335/rlct.v I I i2.845
Para citar este artículo / To reference this article / Para citar este artigo: Pardo Pérez, E., Cavadía Martínez,T. I., \& Cruz Cantero,A. E. (2019). Diversidad genética en humanos mediante polimorfismos de inserción de Alu en la población de San Pelayo, Córdoba (Colombia). Revista Logos Ciencia \& Tecnología, I I (2), 86-92. http://dx.doi.org/I0.22335/rlct. vIlii2.845

\begin{abstract}
RESUMEN
Esta investigación determinó la diversidad genética y la estructura poblacional en poblaciones humanas de San Pelayo, a partir de polimorfismo Alu humano. Las muestras se obtuvieron de adultos no emparentados. El ADN de cada muestra fue extraído mediante el kit de Promega, los marcadores se amplificaron mediante PCR y los amplificados fueron sometidos a electroforesis y visualizados en un transiluminador. Los loci resultaron polimórficos, las frecuencias alélicas mostraron valores heterogéneos. La prueba de Hardy Weinberg indicó que la población no estaba en equilibrio. También se detectó baja heterocigosidad y alto índice de fijación y los marcadores utilizados resultaron apropiados para la evaluación de la diversidad genética. La población presentó baja diversidad y alto nivel polimórfico.
\end{abstract}

Palabras clave: Alu, diversidad genética, polimorfismo, heterocigosidad 


\section{SUMMARY}

This research determined the genetic diversity and population structure in human populations in San Pelayo, based on human Alu polymorphism. The samples were obtained from unrelated adults. The DNA of each sample was extracted using the PROMEGA kit, the markers were amplified by PCR and the amplified ones were subjected to electrophoresis and visualized in a transilluminator. The loci were polymorphic, the allelic frequencies showed heterogeneous values. The Hardy Weinberg test indicated that the population was not in equilibrium. Low heterozygosis and a high fixation index were also detected and the markers used were appropriate for the evaluation of genetic diversity. The population showed low diversity and a high polymorphic level.

Keywords: Alu, genetic diversity, polymorphism, heterozygosis

\section{SUMÁRIO}

Esta pesquisa determinou a diversidade genética e a estrutura populacional em populações humanas, em San Pelayo, a partir do polimorfismo Alu humano. As amostras foram obtidas de adultos não aparentados. O DNA de cada amostra foi extraído com o kit PROMEGA, os marcadores foram amplificados por PCR e submetidos a eletroforese e visualizados em um transiluminador. Os loci eram polimórficos, as frequências alélicas apresentaram valores heterogêneos. $O$ teste de Hardy Weinberg indicou que a população não estava em equilíbrio. Também foram detectados uma baixa heterozigosidade e um alto índice de fixação e os marcadores utilizados foram adequados para a avaliação da diversidade genética. A população apresentou uma baixa diversidade e um alto nível polimórfico.

Palavras-chave: Alu, diversidade genética, polimorfismo, heterozigosidade

Los elementos móviles pueden constituir aproximadamente el $45 \%$ del genoma humano. Entre ellos el grupo más abundante lo compone la familia de elementos Alu. Las inserciones de Alu son marcadores bialélicos que se definen por la ausencia y presencia de unas secuencias de aproximadamente $300 \mathrm{pb}$, que con unas variaciones más o menos importantes derivan del gen 7SL RNA, el cual forma parte del complejo ribosomal por retrotransposición. Son especialmente interesantes en antropogénica por aparecer hace 65 millones de años y encontrarse con el origen y la expansión de los primates (Batzer \& Deininger, 2002). La mayoría de las inserciones Alu son recientes, por lo que en general son polimórficas, pudiendo tomar diferentes valores de frecuencias en distintas poblaciones humanas (Stoneking et al., 1997). Es altamente improbable que la misma inserción Alu ocurra dos veces independientemente en el mismo lugar, por lo que se consideran eventos mutacionales únicos (Batzer \& Deininger, 1994).A nivel mundial se han hecho diferentes reportes de los elementos Alu en diferentes poblaciones, Novick et al. (1998) comparó cinco inserciones Alu en treinta poblaciones de las cuales veinticuatro fueron del continente americano y seis de otras partes del mundo, reportando que las relaciones entre las poblaciones tenían una correlación con la distribución geográfica dentro del continente americano, lo que confirma la sensibilidad con que los marcadores Alu permiten comparar y asociar poblaciones. En Colombia, factores geográficos, climáticos e históricos han contribuido a los patrones de variación genética humana vistos en la actualidad y a nivel mundial. Por ejemplo, los procesos de población asociados a la colonización, los periodos de aislamiento geográfico, la endogamia socialmente reforzada y la selección natural todos han afectado las frecuencias de alelos en ciertas poblaciones (Jorde, Bamshad \& Rogers, 1998; Jorde \& Wooding, 2004). Sin embargo, la actualidad de nuestra ascendencia común y el flujo continuo de genes entre los grupos humanos han limitado la diferenciación genética en nuestra especie (Jorde \& Wooding, 2004).

Las poblaciones del Caribe colombiano se caracterizan por ser híbridas o mezcladas, se tiene un aporte genético particular de los diferentes grupos amerindios, indígenas, afrodescendientes y europeos llegados de España y 
Portugal que conforman cada una de las poblaciones de Colombia (Gutiérrez, 1980). Tanto en Colombia como en el departamento de Córdoba se presenta un alto mestizaje y se desconoce su diversidad genética aún más en trabajos relacionados con los elementos Alu, con excepción del reporte de las estimaciones de mezclas genéticas por elementos Alu en poblaciones afrocolombianas y mestizas en Antioquia, donde se determinaron los procesos de mezclas que ocurren en las poblaciones de América Latina al examinar los perfiles genéticos de dos grupos étnicos de Antioquia (Gómez et al. 2010). En el municipio de San Pelayo hay un gran mestizaje, debido a la colonización de extranjeros españoles, mestizos, blancos y negros. Sin embargo, en este municipio ha aparecido en muchas generaciones el cruzamiento entre familias, lo que hay traído un problema que afecta como tal el deterioro de la diversidad genética en las próximas generaciones. Por lo tanto, en esta investigación se determinó la diversidad genética y la estructura poblacional en humanos de San Pelayo, utilizando cinco polimorfismos de inserción Alu.

\section{Materiales y métodos}

Área de estudio. Las 40 muestras fueron colectadas así: 20 en la subpoblación San-Pelayo Urbano ( $8^{\circ} 57^{\prime} 28^{\prime \prime} \mathrm{N}, 75^{\circ}$

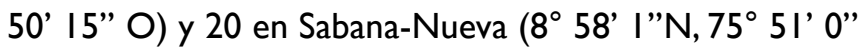
W) en el departamento de Córdoba, Colombia.

Obtención de muestras. Las muestras requeridas para el análisis genético se obtuvieron de adultos voluntarios no emparentados entre sí, residentes estables de la población del estudio, desde al menos tres generaciones. Se recolectaron $2 \mathrm{ml}$ de saliva por cada individuo en dos tubos eppendorf de I,5 ml, después se adicionó solución de lisis celular $500 \mu$ l; los participantes en el estudio firmaron el respectivo consentimiento informado. Posteriormente, las muestras fueron almacenadas en una cava con una temperatura de $4^{\circ} \mathrm{C}$ para ser trasladadas hasta el laboratorio de genética de la Universidad de Córdoba.

Aspectos éticos. Este trabajo cuenta con la aprobación del comité de ética para investigación en humanos de la Facultad de Ciencias de la Salud de la Universidad de Córdoba.

Extracción de $A D N$. Para la extracción de las 40 muestras de ADN se utilizó el kit de extracción y purificación de Wizard Genomic DNA Purification (Promega) y se siguieron las recomendaciones del fabricante sin modificaciones. La pureza y la concentración del ADN fue determinada utilizando I $\mu \mathrm{L}$ de la muestra en un espectrofotómetro Nanodrop 2000® (Thermo Scientific, Waltham, MA, EE. UU.) con relaciones de absorbancia A260/A280 nm y A260/A230 nm. La integridad del ADN se determinó de manera visual mediante electroforesis en geles de agarosa al $1.0 \%$, con un voltaje de $120 \mathrm{~V}$ por $2 \mathrm{~h}$ en buffer TBE IX (Tris-HCI 500 mM, ácido bórico 60 mM y EDTA 83 mM) y agente de tinción GelRed IX. El gel se visualizó en un transiluminador Bio-Imagen System 312 nm, Neve Yamin, Israel.

Amplificación por PCR. Los cebadores Alu: DI (Batzer et al., 1995), FXIII, APO y PV92 (Batzer et al., I994) y ACE (Tired et al., 1992) se observan en la tabla I, fueron amplificados mediante la técnica de la PCR.

La técnica de la PCR (tabla 2) se realizó en un volumen total de $25 \mu \mathrm{l}$ que contenía $0,25 \mu \mathrm{l}$ de Taq polimerasa (Thermo Scientific) (Uklam-EE. UU.), I,25 $\mu \mathrm{l}$ de cada primer (forward y reverse), $0,5 \mu \mathrm{l}$ de dNTPs a $10 \mathrm{mM} / \mu \mathrm{l}, 2,5 \mu \mathrm{l}$ de buffer de reacción a IOX, I,25 $\mu \mathrm{l}$ de $\mathrm{MgCl} 2,5 \mu \mathrm{l}$ de ADN y agua estéril hasta alcanzar el volumen final. La reacción de PCR se realizó en un termociclador Bioard TI00 (Los Ángeles, EE. UU.) mediante la técnica PCR Tochdown.

Tabla I. Características de las secuencias de los elementos Alu

\begin{tabular}{|c|c|c|c|c|c|}
\hline Locus & LC & SF & Secuencia (5'- 3') & $\mathbf{T}^{\circ} \mathbf{C}$ & Tamaño del fragmento (pb) \\
\hline$A C E$ & $17 q 23$ & Ya5 & $\begin{array}{l}\text { F: CTGGAGACCACTCCCATCCTTTCT } \\
\text { R: GATGTGGCCATCACATTCTCAGAT }\end{array}$ & 58 & $490 / 190$ \\
\hline PV92 & $16 q 23.3$ & Ya5/Ya8 & $\begin{array}{l}\text { F:AACTGGGAAAATTTGAAGAGAAAGT } \\
\text { R:TGAGTTCTCAACTCCTGTGTGTTAG }\end{array}$ & 54 & $437-122$ \\
\hline$A P O$ & I I q23.3 & Ya5 & $\begin{array}{l}\text { F:AAGTGCTGTAGGCCATTTAGATTAG } \\
\text { R:AGTCTTCGATGACAGCGTATCAGA }\end{array}$ & 52 & $443 / 129$ \\
\hline FXIII & Iq31-q32.I & Ya5 & $\begin{array}{l}\text { F:TCAACTCCATGAGATTTTCAGAAGT } \\
\text { R: CTGGAAAAAATGTATTCAGGTGAGT }\end{array}$ & 56 & $700-410$ \\
\hline DI & $3 q 26.32$ & Yb8 & $\begin{array}{l}\text { F:TGCTGATGCCCAGGGTTAGTAAA } \\
\text { R:TTTCTGCTATGCTCTTCCCTCTC }\end{array}$ & 65 & $670 / 333$ \\
\hline
\end{tabular}

LC: localización cromosómica; SF: subfamilia, $\mathrm{T}^{\circ} \mathrm{C}$ : temperatura de hibridación 
Tabla 2. Ciclos PCR realizados para la amplificación de 5 inserciones Alu

\begin{tabular}{ccc|} 
Temperatura & Tiempo & Número de ciclos \\
\hline $95^{\circ}$ & $1-3 \mathrm{~min}$. & $\mathrm{I}$ \\
$95^{\circ}$ & $30 \mathrm{seg}$. & 39 \\
$52^{\circ}-65^{\circ}$ & $30 \mathrm{seg}$. & \\
$72^{\circ}$ & $1 \mathrm{~min}$. & 1 \\
\hline $72^{\circ}$ & $5-15 \mathrm{~min}$. & 1 \\
\hline
\end{tabular}

Visualización y cuantificación del ADN. Los amplificados de la PCR fueron sometidos a electroforesis en gel de agarosa al $2 \%$, a 100 voltios durante 40 minutos, estos resultados fueron visualizados bajo luz ultravioleta en una cámara de (Cleaver Scientific) (Londres, UK). Además, en el gel de agarosa se añadió un marcador de peso molecular de separación 500 pb, con el objeto de identificar el tamaño de las bandas de la PCR (figura I).

Análisis estadístico. Para la determinación de los parámetros de diversidad genética: frecuencias alélicas, heterocigosidad observada y esperada, equilibrio de Hardy-Weinberg, y estadísticos $\mathrm{F}$ de Wright se utilizó el software GenAlex 6.502 (Peakall \& Smouse, 20I2).

\section{Resultados}

En la figura I se puede observar la electroforesis de 38 muestras para el cebador ACE (Pb 490/190), donde se pueden observar homocigotos con la inserción: individuos I,8, I3, 15, 16, 17, 21, 22, 23, 30,31, 34, 35, heterocigotos: individuos 2, 3, 4, 5, 6, 7, I2, I4, I8, 19, 24, 26, 28 y homocigotos sin inserción: individuos 9, 10, II, 23, 27, 32, 33.

En la tabla 3 se observan los valores de las frecuencias alélicas obtenidas para los marcadores Alu, en las dos subpoblaciones estudiadas, todos los loci analizados resultaron polimórficos. Al determinar las frecuencias alélicas para cada subpoblación (tabla 3) se obtuvo que para San Pelayo urbano y Sabana-Nueva, las inserciones DI, PV92 y APO muestran los mayores valores $y$ las menores frecuencias se encontraron para los marcadores ACE y FXIII respectivamente. La frecuencia alélica promedio resultó mayor en San Pelayo Urbano $(0,425)$ que en Sabana-Nueva $(0,324)$.

Tabla 3. Frecuencias alélicas de 5 marcadores Alu

\begin{tabular}{|cccc|}
\hline Loci & Alelo & San-Pelayo Urbano & Sabana-Nueva \\
\hline ACE & 490 & 0,125 & 0,375 \\
\hline APO & 409 & 0,500 & 0,521 \\
\hline DI & 670 & 0,636 & 0,227 \\
\hline FXIII & 700 & 0,231 & 0,200 \\
\hline PV92 & 443 & 0,636 & 0,300 \\
\hline Promedio & 542,4 & 0,425 & 0,324 \\
\hline
\end{tabular}

En la tabla 4 los valores obtenidos para el equilibrio de Hardy-Weinberg en las dos subpoblaciones mostraron ausencia de valores equilibrio de Hardy-Weinberg

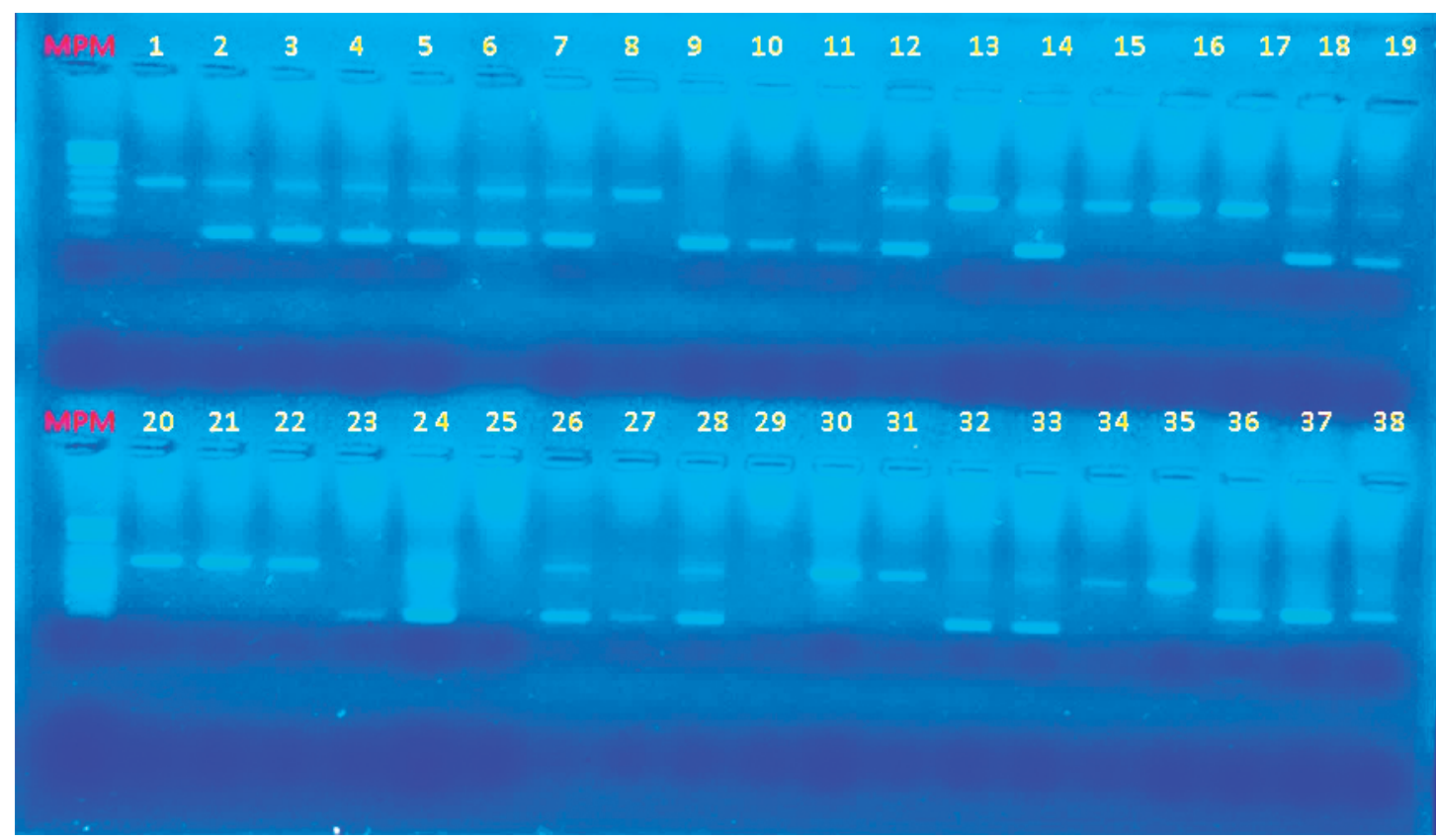

Figura I. Electroforesis de productos PCR de la inserción ACE (Pb 490/190) 
$(p<0,05)$ para los cinco loci analizados. La heterocigosidad observada $(\mathrm{Ho})$ mostró valores entre 0,042 y 0,000. Los valores de heterocigosidad esperada $(\mathrm{He})$ varían entre 0,219 para la subpoblación de San Pelayo Urbano hasta 0,500 y para la subpoblación de Sabana-Nueva se observaron valores de 0,320 y 0,499 . El índice de fijación indica un valor de I,000 en la subpoblación de San-Pelayo urbano en general, pero en la subpoblación de SabanaNueva solo el loci APO tuvo un valor de 0,917.

Tabla 4. Parámetros genéticos calculados en la zona de estudio

\begin{tabular}{|c|c|c|c|c|c|}
\hline Población & Loci & $\mathbf{P}$ & Ho & $\mathrm{He}$ & $\mathbf{F}$ \\
\hline \multirow[t]{5}{*}{ San Pelayo Urbano } & $A C E$ & $0,005^{* *}$ & 0,000 & 0,219 & 1,000 \\
\hline & APO & $0,000 * * *$ & 0,000 & 0,500 & 1,000 \\
\hline & DI & $0,00 I^{* *}$ & 0,000 & 0,463 & 1,000 \\
\hline & FXIII & $0,000 * * *$ & 0,000 & 0,355 & 1,000 \\
\hline & PV92 & $0,00 \mathrm{I} * *$ & 0,000 & 0,463 & 1,000 \\
\hline \multirow[t]{5}{*}{ Sabana-Nueva } & $A C E$ & $0,000 * * *$ & 0,000 & 0,469 & 1,000 \\
\hline & $A P O$ & $0,000 * * *$ & 0,042 & 0,499 & 0,917 \\
\hline & DI & $0,000 * * *$ & 0,000 & 0,351 & 1,000 \\
\hline & FXIII & $0,000 * * *$ & 0,000 & 0,320 & 1,000 \\
\hline & PV92 & $0,000 * * *$ & 0,000 & 0,420 & 1,000 \\
\hline
\end{tabular}

P: equilibrio Hardy-Weinberg (** $\mathrm{p}<0,0 \mathrm{I}$, *** $\mathrm{p}<0,00 \mathrm{I}$ ), Ho: heterocigosidad observada, He: heterocigosidad esperada, F: índice de fijación

En la tabla 5 los valores de $F_{S T}$ varían de 0,00 en el marcador $A P O$ a 0,17 para el locus $D I$. En el caso de las $F_{15}$ y $F_{1 T}$ mostraron valores de 0,958 a I,000.

Tabla 5. Valores de los estadísticos $\mathrm{F}$ para cada marcador en la población global

\begin{tabular}{cccc} 
& $F_{S T}$ & $F_{I T}$ & $F_{I S}$ \\
\hline ACE & 0,083 & 1,000 & 1,000 \\
APO & 0,000 & 0,958 & 0,958 \\
DI & 0,171 & 1,000 & 1,000 \\
FXIII & 0,001 & 1,000 & 1,000 \\
PV92 & 0,114 & 1,000 & 1,000 \\
Promedio & 0,074 & 0,992 & 0,992 \\
\hline
\end{tabular}

\section{Discusión}

Los cinco marcadores analizados resultaron polimórficos en las dos subpoblaciones, las inserciones Alu mostraron valores heterogéneos dentro del rango de las frecuencias alélicas. Se observa una afinidad con las frecuencias alélicas de las poblaciones europea-americana, y la africanaamericana (Watkins et al., 200I). Los valores promedios de las frecuencias alélicas de San Pelayo Urbano fueron más cercanos a las reportadas en Rongmei, Inpui y Kuki y las de Sabana Nueva a las de Zeme Liangmai (Kshatriyaa, Panmeib \& Kameihc, 2019).

La heterocigosidad observada $(\mathrm{Ho})$ presentó valores bajos en comparación con lo reportado para las poblaciones de América y de otros lugares del mundo (Stoneking et al., 1997) lo cual pudo ser ocasionado por la endogamia. Los valores de la heterocigosidad esperada $(\mathrm{He})$ fueron superiores debido a la homogeneidad de las frecuencias alélicas en los marcadores, por tanto, lo que se espera en la siguiente generación es un número de homocigoto mucho más altos que de heterocigotos, estos resultados de heterocigosidad esperada se asemejan a lo reportado para las poblaciones amerindias, las poblaciones africanas y las poblaciones asiáticas (Antúnez et al., 2002; Battilana et al., 2006).

Los cinco marcadores no estuvieron en equilibrio de Hardy-Weinberg para las subpoblaciones, esto pudo deberse a diferentes eventos como el efecto Wahlund, suceso que no afecta por igual a todo el genoma y produce un aumento de homocigotos y una disminución de heterocigotos, lo cual está en concordancia con los resultados obtenidos y también por la endogamia originada por un aislamiento de las dos subpoblaciones: en la historia de San Pelayo, durante el proceso de colonización entre los años 1690 y I72I, se reporta que las familias del municipio se casaban entre ellas (Fortich, 2014) y para el caso de Sabana Nueva en su reseña histórica se afirma que esta fue fundada por españoles que llegaron vía fluvial a finales del siglo XVIII, donde al igual que en San Pelayo, predominó un entrecruzamiento entre las familias fundadoras para poder mantener su abolengo en las siguientes generaciones, por esto es común encontrar apellidos que predominan como Petro, Hernández, Doria y Cantero, considerados en los estudios antropológicos como los primeros pobladores de esta localidad y determinantes para el predominio del color de piel de estos pobladores (Fortich, 20I4). La ausencia del equilibrio de Hardy Weinberg obtenida en esta investigación, fue similar a los resultados reportados en poblaciones amerindias colombianas para marcadores Alu (Novick et al., 1998).

Los valores obtenidos para el índice de fijación mostraron que las dos subpoblaciones presentaron un aislamiento genético. Respecto a los estadísticos $\mathrm{F}$ se observó que en los valores de $F_{S T}$ mostraron valores cercanos a cero en casi todos los marcadores, indicando que las dos subpoblaciones se comportan como una sola población; 
estos resultados son menores a lo reportado en poblaciones de Europa, África y afrocolombianas (Gómez et al., 20I0, Terreros et al., 2009). Además, la diversidad genética obtenida en el presente estudio puede atribuirse a variaciones individuales dentro de las poblaciones, ya que solo el $7,4 \%$ de la diversidad genética total proviene de la variación entre las poblaciones $\left(\mathrm{F}_{\mathrm{ST}}\right)$.

Al mismo tiempo, las $F_{\text {IS }} Y F_{I T}$ ratifican el déficit de heterocigoto, estos resultados se dan por consecuencia de apareamientos consanguíneos. Estos valores obtenidos no poseen similitud con las poblaciones brasileñas (Cotrim et al., 2004).

\section{Conclusiones}

La población de San Pelayo, Córdoba (Colombia) presentó una baja diversidad genética, $y$ un alto nivel polimórfico en todos los elementos Alu. El exceso de homocigotos que hay en toda la población es producido por la endogamia, lo cual aumenta la posibilidad de pérdida de los alelos para las siguientes generaciones, en consecuencia, esto llevaría a la disminución de la diversidad genética a lo largo del tiempo.

\section{Referencias}

Antúnez, G., Antúnez, P., Papilha, P., Hammer, S., Yunis, M.,Yunis, J. J., Damodaran, E. J., Martinez De Pancorbo, C., Caeiro, M., Puzyrev,J.L.,\&Herrera,V.P.(2002).Phylogenetics of worldwide human populations as determined by polymorphic Alu insertions. Electhrophoresis, 23, 3346-3356. http://dx.doi. org// 0.1002/I522-2683(2002/0)23:19<3346::AIDELPS3346>3.0.CO;2-J

Battilana, J., Fagundes, N. J. R., Heller, A., Goldani, A. Freitas, L. B., Tarazona, E., \& Bonatto, S. L. (2006). Alu insertion polymorphisms in Native Americans and related Asian populations. Annals of Human Biology, 33(2), I42-160. http:// dx.doi.org//0.1080/030|4460500487347

Batzer, Ma., \& Deininger P. L. (1994). A human-specific subfamily of Alu sequences. Genomics, 9, 48I-487. http:// dx.doi.org/I0.1016/0888-7543(9I)904I4-A

Batzer, M. A., Stoneking, M., Alegria-Hartman, M., Bazan, H., Kass, D. H., Shaikh,T.H., Novick, G. E., loannou, P.A., Scheer, W.D., Herrera, R. J., \& Deininger, P. L. (1994).African origin of human-specific polymorphic Alu insertions. Proceedings of the National Academy of Sciences USA 9I, I 2288- 12292. http://dx.doi.org// 0.1073/pnas.91.25.12288

Batzer, M. A., Rubin, C. M., Hellman-Blumberg, U., AlegriaHartman, M., Leeflang, E. P., Stern, J. D., Bazan, H.A., Shaikh, T. H., Deininger, P. L., \& Schmid, C.W. (1995). Dispersion and insertion polymorphism in two small subfamilies of recently amplified human Alu repeats. Journal Molecular Biology, 247, 4/8-427. http://dx.doi.org//0.1006/ jmbi. 1994.0150

Batzer, M.A., \& Deininger, P. L. (2002). Alu repeats and human genomic diversity. Nature Reviews Genetics, 3, 370-379. http://dx.doi.org// 0.1038/nrg798

Cotrim, N. H., Auricchio, M. T., Vicente, J. P., Otto, P. A., \& Mingroni-Netto, R. C. (2004). Inserciones polimórficas de Alu en seis poblaciones brasileñas derivadas de África. American Journal of Human Biology, 65, 234-24I. http:// dx.doi.org// 0.1002/ajhb.20024

Fortich, D.W. (20I4). Sabana-Nueva, tradición y cultura, historia documental de Córdoba. Montería: Domus Libri.

Gómez, L., Sánchez, M. A., Pérez, A. M., García, S., Builes, J. J., Bravo, M. L., Pancorbo, M. M., \& Peña, J. A. (20I0). Genetic admixture estimates by Alu elements in Afro-Colombian and Mestizo populations from Antioquia, Colombia. Annals of Human Biology, 37(4), 488-500. http://dx.doi. org/I0.3109/03014460903433810

Gutiérrez, I. (1980). Historia del negro en Colombia. Bogotá: Editorial Nueva América..

Jorde, L. B., Bamshad, M., \& Rogers, A. R. (1998). Using mitochondrial and nuclear DNA markers to reconstruct human evolution. BioEssays, 20, 126-136. http://dx.doi. org/ I0.1002/(SICl) I 52 I- I878(I99802)20:2<126::AIDBIES5>3.0.CO;2-R

Jorde, L. B., \& Wooding, S. P. (2004). Genetic variation, classification and 'race'. Nature Genetics, 36(I I), S28-33. http:// dx.doi.org//0.1038/ngl435

Kshatriyaa, G. K., Panmeib, T., \& Kameihc, G. (20/9). Alu insertion-deletion polymorphisms in the Tibeto-Burman speaking tribal groups of Manipur, North-East India. Gene Reports, 15, I-5 http://dx.doi.org/10.1016/j.genrep.2019.100372

Novick, G., Novick, C., Stokening, M., Batzer, M., Yunnis, E., \& Deinnger, P. (1998). Polymorphic Alu insertion in the Asian origin of Native American population. Human Biology, 70 (I), 23-39

Peakall, R., \& Smouse, P. E. (20I2). GenAlex 6.502: análisis genético en Excel. Software de genética poblacional. Bioinformatics, 8, 2537-2539. http://dx.doi.org//0.1093/ bioinformatics/bts 460

Stoneking, M., Fontius, J., Clifford, S., Soodyall, H., Arcot, S., Saha, N., Jenkins, T., Tahir, M., Deininger, P., \& Batzer, M.A. (1997). Alu insertion polymorphisms and human evolution: evidence for a larger population size in Africa. Genome Research, 7, |06|-107| 
Terreros, M., Sánchez, M. A., Novick, G., Harlette Lacau, H., Lowery, C., Regueiro, M., \& Herrera, R. (2009). Insights on human evolution: An analysis of Alu insertion polymorphisms. Journal of Human Genetics, 54, 603-6II. http://dx.doi.org/10.1038/jhg.2009.86

Tiret, L., Rigat, B.,Visvikis, S., Breda, C., Corval, P., Cambien, F., \& Soubrier, F. (1992). Evidence, from combined segregation and linkage analysis, that a variant of the angiotensin l-converting enzyme (ACE) gene controls plasma ACE levels. American Journal of Human Genetics, 5I, 197-205.
Watkins, W. S., Ricker, C. E., Bamshad, M. J., Carroll, M. L., Nguyen, S. V., Batzer, M. A., Hapending, H. C., Rogers, A. R., \& Jorde L. B. (200I). Patterns of ancestral of Aluinsertion and restriction-site polymorphisms. American Journal of Human Genetics, 68, 738-752. http://dx.doi. org/I0.1086/318793 